\title{
TIDAL EFFECTS ON SPHERICAL STELLAR SYSTEMS
}

\author{
D. W. KEENAN and K. A. INNANEN \\ Physics Dept., York University, Downsview, Ontario, Canada
}

Many self-gravitating stellar systems are satellites of larger galaxies and must therefore be subjected to the tidal field of the parent system. Examples are the globular clusters and dwarf elliptical galaxies, which are satellites of our Galaxy. Most previous studies of tidal effects have been highly simplified, e.g. clusters in circular planar galactic orbits (Bok, 1934), or have assumed that the tidal field acts to limit the size of a star cluster without any effects on its internal structure or stability (Spitzer and Shapiro, 1972; Spitzer and Thuan, 1972).

To determine the effects of a realistic time varying tidal field on a stellar system where binary encounter processes are unimportant, we computed the orbits of a large and representative sample of test particles in the field of a spherically symmetric smooth model of a star cluster which, in turn, moved in its own orbit around a model galaxy. The effect of the tidal fields on the stellar orbits was investigated by observing the evolution of the stars in energy $E$ and angular momentum $h . E$ and $h$ are, of course, conserved quantities for an isolated spherically symmetric field. Three systems were tested. Two involved a point mass galaxy with the cluster in an elliptical orbit about it. The third system used a rotationally symmetric model of a disk halo galaxy. The mass model was constructed to fit the mass distribution and kinematics of our Galaxy (Innanen, 1973). This system was an analogue for a 'typical' halo globular cluster in our Galaxy.

The three body equations of motion were integrated using an IBM 370/75 system and a fast double precision algorithm kindly supplied by Dr F. T. Krogh of J. P. L. (Krogh, 1969, 1971).

The main conclusion reached in these numerical experiments is that star clusters rotating in a retrograde sense compared with their galactic orbital motion, are much more stable in a tidal field than clusters with either direct rotation or no rotation. Observational support is given to this by measurement of the rotation of $\omega$ Centauri (Harding, 1965; Keenan et al., 1973) and of M13 (Griffin, 1972a, b, 1974). The most stable stellar orbits are low-eccentricity, low inclination retrograde orbits. The least stable are similar direct orbits. For the case of a disk model galaxy, the stellar orbits inclined at large angles to the galactic plane are also highly perturbed due to the large field gradients across the disk in the vertical direction.

The galactic orbits of the stars which escaped from the model cluster due to the tidal interaction were also computed. The escapers were grouped into two 'clumps' in energy-angular momentum phase space. These clumps are separated from each other and from the point occupied by the parent cluster in orbital energy and angular momentum phase space. One 'clump' has lower energy and angular momentum than the cluster while the other has higher. 
This clumping of the escapers' orbits may be explained by the existence of 'quasiintegrals' analogous to the Jacobi integral in the restricted problem of three-bodies (Szebehely, 1967), which restricts the motion of the body to certain regions of phase space.

A 'randomizing' action of the tidal field on the energies and angular momenta of the bound stars was also noted. This would be analogous to the 'shock induced relaxation' discussed by Spitzer and Chevalier (1973).

This work will be published in greater detail elsewhere (Keenan and Innanen, 1975).

\section{Acknowledgements}

We would like to thank Dr I. R. King for many useful discussions. This research was supported by the National Research Council of Canada.

\section{References}

Bok, B. J.: 1934, Harvard College Obs. Circular 384.

Harding, G. A.: 1965, Roy. Obs. Greenwich-Cape Bull. No. 99, 14S.

Griffin, R.: 1972a, Observatory 92, 29.

Griffin, R.: 1972b, Quart. J. Roy. Astron. Soc. 13, 442.

Griffin, R.: 1974, Private Communication.

Innanen, K. A.: 1973, Astrophys. Space Sci. 22, 393.

Keenan, D. W., Innanen, K. A., and House, F. C.: 1973, Astron. J. 78, 173.

Keenan, D. W. and Innanen, K. A.: 1975, Astron. J. (in press).

Krogh, F. T.: 1969, T.U. Doc. No. CP-2308, J.P.L., Pasadena.

Krogh, F. T.: 1971, T.U. Doc. No. CP-2586, J.P.L., Pasadena.

Spitzer, L. and Shapiro, S. L.: 1972, Astrophys. J. 173, 529.

Spitzer, L. and Thuan, T. X.: 1972, Astrophys. J. 175, 31.

Spitzer, L. and Chevalier, R. A.: 1973, Astrophys. J. 183, 565.

Szebehely, V.: 1967, Theory of Orbits, Academic Press, New-York.

\section{DISCUSSION}

Freeman: Could you say what fraction of escapers are lost during passage through the galactic plane compared with other escapers.

Innanen: We have not looked at this question quantitatively, but we hope to do so.

Freeman: Your comment about a quasi-Jacobi-integral is very likely correct, because the time for a star to cross the cluster is short compared to the other time-scales involved.

Miller: Are there effects due to repeated perigalacticon passage - some stars that are set up on one passage that escape on next passage? Do some become more stable?

Innanen: Yes. Yes.

Baldwin: Are there any significant changes in the net angular momentum of the dwarf spheroidal system during the passages?

Innanen: Probably not.

Gott: $\omega$ Cen is the most massive globular cluster and also has the most elliptical isophotes. It would be your contention that it is in retrograde rotation?

Innanen: Yes. The clusters with predicted greater stability are those whose rotation is in the opposite sense to their sense of revolution.

Gott: Also would escape of the direct orbit stars be expected to leave all globular clusters with a net retrograde rotation?

Innanen: I don't know; the escaping stars would escape mainly from the outer parts of these systems. 
The dense inner parts which are the ones that are really observed are not very strongly affected by these computations. Those parts which are interesting from the escaping or non escaping point of view are really well out in the halo and therefore are difficult to establish observationally, at least at the present time.

Lynden-Bell: I think $\omega$ Cen has a retrograde galactic orbit so we must get straight the meanings of retrograde and direct in this discussion.

Innanen: Same answer as given to Gott.

King: Is it not possible that the stability of retrograde orbits is due to the presence of a pseudo-integral? This seemed to be the case in the orbital studies of the restricted three-body problem by Henon and by Jefferys. It appeared there that some bodies that were not bound were nevertheless bounded, by the existence of a pseudo-integral.

Innanen: No. We have not examined our results from the Hénon phase-space point of view.

Hénon: In numerical studies of the restricted three-body problem, one finds exactly the same result, namely that retrograde orbits are stable at larger distances than direct orbits. In celestial mechanics also this effect is well known: cf. for instance the fact that the four outer satellites of Jupiter are on retrograde orbits.

Kalnajs: Although the orbital periods of your clusters around the galaxy are much larger than the orbital periods of the stars in the cluster, the rate of change of the tidal field as seen by star crossing the galactic plane seems fast. How does the time-scale of this change compare to the periods of the preescapers?

Innanen: The time of application of the impulsive force when crossing the plane is, in effect, comparable to the orbital period of the star in the cluster. It does however depend considerably on the inclination of the cluster orbit to the galactic plane, which is sometimes high, and sometimes quite low. 